\title{
CONDICIONES ALIMENTARIAS DE LOS MAYAS MACEHUALES DE Quintana Roo
}

\author{
Virginia Ivonne Sánchez Vázquez \\ Erin Ingrid Jane Estrada Lugo \\ Ana Minerva Arce Ibarra \\ Reynaldo Germán Martínez Velasco
}

\begin{abstract}
Resumen: Las políticas alimentarias ofrecen una visión oficial sobre las condiciones de la alimentación en México, y específicamente en el México rural indígena, pero a partir de un estudio de caso con los grupos mayas macehuales de Quintana Roo, se muestra el contexto de confrontación donde las comunidades despliegan sus pautas de reproducción social, ciertamente en desventaja estructural, pero no de manera pasiva, sino creando y recreando opciones bajo su marco cultural situado.
\end{abstract}

Palabras clave: Desarrollo, políticas alimentarias, grupos domésticos, mediaciones culturales.

Enviado a dictamen: 22 de agosto de 2011

Aprobación: 21 de octubre de 2011

Revisiones: 1

Mtra. Virginia Ivonne Sánchez Vázquez, candidata a doctora en Ciencias en Ecología y Desarrollo Sustentable, por El Colegio de la Frontera Sur, San Cristóbal de Las Casas, Chiapas. Temas de especialización: Economía campesina e indígena, formas de organización social, uso y manejo de los recursos naturales; grupos domésticos; derechos, salud sexual y reproductiva. Correo electrónico: vivonne@hotmail.com.

Dra. Erin Ingrid Jane Estrada Lugo, doctora en Antropología Social. Labora en El Colegio de la Frontera Sur, San Cristóbal de Las Casas, Chiapas. Temas de especialización Organización social y apropiación del territorio en el uso de los recursos naturales en las sociedades campesinas indígenas; Familia, Grupo Doméstico y Parentesco. Correo electrónico: eestrada@ecosur.mx.

Dra. Ana Minerva Arce Ibarra, doctora en Filosofía. Labora en El Colegio de la Frontera Sur, Unidad Chetumal. Temas de especialización: Pesquerías de pequeña escala. Valoración económica de recursos, economía ecológica y manejo comunitario de recursos naturales renovables, Interdisciplinariedad. Correo electrónico: aarce@ecosur.mx. Dr. Reynaldo Germán Martínez Velasco, doctor en Ciencias Sociales. Labora en El Colegio de la Frontera Sur, San Cristóbal de Las Casas, Chiapas. Temas de especialización: Pobreza y Marginación; Migración y cambio sociocultural; Impacto de la emigración de chiapanecos en los lugares de origen; y Política migratoria en la Frontera Sur de México. Correo electrónico: gmartine@ecosur.mx.
Abstract: In Mexico food policies offer an official view on the conditions of food supply, specifically on rural and indigenous settings, but based on a case study with the indigenous Mayas Macehuales of Quintana Roo, it is shown that the context of confrontation in which communities display patterns of social reproduction, are indeed in structural disadvantage, but not passively. Rather, it is through creating and recreating cultural options under their own framework set.

Keywords: Development, food policy, domestic groups, cultural mediations.

\section{Introducción}

L a alimentación humana, en tanto hecho biológico, social y cultural, requiere un análisis integral _ para promover su redefinición, con respecto a los conceptos y prácticas económicas, ambientales, sociales y políticas, propias de cada contexto. ${ }^{1}$ Esto es factible si se concibe la alimentación como un "hecho social total" (Mauss, 1925), integrador e integrado, y simultáneamente como un fenómeno de carácter interdependiente.

El análisis antropológico de las políticas alimentarias, considera el "hecho etnográfico" no sólo como la multiplicidad de "prácticas y conocimientos locales", sino también en interrelación con el marco de las actuales formas de atención (intervención) 
alimentaria. Las políticas alimentarias deben implicar una ética disciplinaria y social, que valore a "los otros y a sus realidades", a partir de su marco cultural situado (Escobar, 2005).

El objetivo de este trabajo es explicar los problemas alimentarios como resultado de la configuración de una estructura que estima a ciertas poblaciones "afectadas" tanto por condiciones biológicas (en pobreza alimentaria) como por condiciones sociales (dependientes o subsidiadas) específicas. Para ello, en este escrito se expone como estudio de caso al Ejido de X-Hazil Sur y Anexos, perteneciente a la región maya macehual de Quintana Roo, México.

Implementar las políticas de atención alimenticia apropiadas, requiere un análisis exhaustivo en torno a la pregunta de icómo promover la interacción entre la urgencia alimenticia y la aplicación de iniciativas políticas al respecto, o en otras palabras, entre la intrincada realidad y las iniciativas gubernamentales, y que a su vez, estas series de interacciones sean culturalmente respetuosas, socialmente pertinentes y ambientalmente adecuadas?

En la siguiente sección se presentan las perspectivas teóricas que permiten analizar las políticas públicas en torno al hecho alimentario, para después contrastarlas con los conflictos, resistencias y creatividades de las respuestas locales.

Una de las aproximaciones esboza el examen de la definición de las políticas públicas en interacción con el Estado, en relación con los aspectos alimentarios. Otra se enfoca en una perspectiva histórica, que recupera una serie de puntos de vista oficiales sobre las políticas y los discursos en torno a esta problemática utilizados en las acciones administrativas; aproximación que culmina con algunos aportes alternos.

Como enlace entre las dimensiones macro económicas (política pública) y las respuestas locales (inter e intra grupos domésticos), se trabaja la perspectiva intermedia, presentando los indicadores en materia alimenticia que ha elaborado el CONEVAL, ${ }^{2}$ respectivos al municipio de Felipe Carrillo Puerto, Quintana Roo, México.

Al final se documentan las acciones que los grupos domésticos emprenden para contrarrestar el marco de constricciones económicas; acciones que desde lo local, suelen confrontarse para condensarse en pautas culturalmente pertinentes y diversas.

\section{Las políticas públicas, definiciones y alcances}

Las condiciones de vida vigentes en México, se derivan de la alineación de las políticas públicas hacia las determinaciones de ajuste estructural, que priorizan la cuadratura de los indicadores macroeconómicos, a costa del bienestar de las personas. En este sentido, el gasto para combatir la pobreza ha sido excluyente, asistencialista y ha funcionando solamente como un paliativo, sin proponerse resolver sus causales (http:// www.voltairenet.org/articlel467l4.html).

Aunque México asumió compromisos como la firma de tratados comerciales (TLCAN), ${ }^{3}$ pese a las diferencias económicas inherentes entre los países involucrados, y sin establecer fondos compensatorios consecuentes, las metas previstas no se lograron, y la perspectiva social dejó de ser prioridad desde mediados de la década de 1980, resultando de ello la reducción del presupuesto al sector primario, desde dicha década hasta el presente (González y Macías, 2007).

En 1981, el gasto federal en el sector primario representó 1.37\% del Producto Interno Bruto (PIB), cifra baja considerando que dicho sector aportó cerca del 6.5\% anual respectivo. Sin embargo, en 1999, esta asignación decreció hasta sólo un 0.44\%. Otro ajuste estructural fue el cierre y la privatización de empresas paraestatales y organismos de apoyo al sector agropecuario. Desde entonces, el agro mexicano afronta una competencia desigual, interna y externa, que socava su capacidad productiva y crea inseguridad en el contexto global (González y Macías, 2007). 
El debate sobre el contenido y enfoque para analizar las políticas públicas, muestra vertientes sobre el papel de éstas y el papel del Estado. Para Harold Lasswell, implican el conocimiento y proceso de toma de decisiones en el orden público y civil. Mientras que para Thoenig y Mény, atienden a la acción de las autoridades públicas en el seno de la sociedad. La diferencia entre ambas definiciones radica en que la última, habla de la autoridad pública ejercida por el Estado, considerando elámbito público como una más de sus partes (Cabrero, 2000: 195).

Con esta última posición, el trabajo del Estado rebasa los intereses de la sociedad civil, y las políticas y programas se definen, ejecutan y evalúan, principalmente, por el gobierno. Por eso Cabrero (2000: 194) resalta la importancia de considerar los referentes institucionales, para entender los sistemas de acción social, particularmente en la política pública, como una vía metodológica para razonar sobre las orientaciones y dinámicas analizadas en el contexto específico, acordes a una realidad histórica.

El autor plantea tres referentes de análisis: a) los globales, en el marco de la ideología dominante; es decir, los del sentido común y las representaciones compartidas; b) los sectoriales, insertos en el marco profesional; yc) los normativos, que regulan los sectores en cuestión, así como los elementos complejos en superposición con las diversas representaciones entre actores, tradiciones, y espacios (Cabrero, 2000).

Coincidiendo con esto, Subirats (1995: 17) enfatiza que al evaluar dichas políticas, se debe analizar el quehacer de las personas al respecto, las implicaciones reales en sus problemas y conocer sus estrategias; de forma que esa información, más el análisis científico, garanticen instrumentos de política pública acertados.

En el mismo sentido, Canto Sáenz (2000) considera la participación de los ciudadanos en el diseño e implementación de las políticas públicas, como un modelo a seguir por las sociedades abiertas, plurales y democráticas.
La política pública en México se vive participando de forma diferente, según el contexto cultural; incluso las diversas historias regionales, pueden explicar diferencias culturales en la vida social y política del país (Canto, 2000). Las políticas públicas deberían atender objetivos concretos de la sociedad, teniendo como resultado planes y programas gubernamentales enfocados hacia el fomento económico progresivo, buscando que los países en desarrollo devengan gradualmente desarrollados, al fortalecer los sistemas de producción y distribución de los bienes y los servicios económicos, sociales, culturales y ambientales (Escobar, 2005).

Pero la ideología neoliberal es el referente de la política agroalimentaria en México, centrada sólo en los indicadores macroeconómicos. Este esquema "de arriba hacia abajo" aplicado por el Estado, incrementó la inseguridad alimentaria, las desigualdades campociudad entre los diferentes sectores socioeconómicos (González y Macías, 2007). Además, se debe mencionar, que no se prioriza el desequilibrio y la degradación ambiental provocados por dichas iniciativas (Canto, 2000).

La rigidez neoliberal en México ha sobrevivido largo tiempo, y es inigualable con cualquier otro país del mundo. Incluso los promotores del ajuste estructural (como el Banco Mundial y el Fondo Monetario Internacional) han criticado sus propias recomendaciones, sugiriendo reorientarlas; no obstante el gobierno mexicano es contundente (Hewitt, 2007). Bajo esta lógica, las personas deben autopromoverse, de manera que, en este contexto, triunfará el más apto. Mientras tanto, el Estado administra la ley y endosa la seguridad nacional, desvinculándose de las demandas sociales (Aguayo, 2002).

De acuerdo con Hewitt (2009) entre los períodos gubernamentales de Luis Echeverría y López Portillo (1970-1982) se buscó reforzar el papel del Estado para impulsar el desarrollo rural y promover la seguridad alimentaria nacional. Sin embargo, con el colapso 
petrolero y la crisis económica de 1982 desaparecieron iniciativas tales como la del Sistema Alimentario Mexicano (SAM). Desde ahí, la visión periférica hacia el agro que ahora supone el acceso a los alimentos sin la producción interna. El argumento es que las ventajas en ciertos renglones productivos prosperan por su propia lógica; mientras que los sectores no competitivos se autoexcluyen por sus condiciones inherentes. La seguridad alimentaria se debe garantizar por la capacidad de compra del gobierno en los mercados mundiales, y no por la producción agropecuaria interna (Hewitt, 2009).

El modelo neoliberal carece de argumentos que justifiquen la nula atención al desarrollo económico rural (innovaciones y diversificaciones productivas). Además dicha tarea se ha canalizado a la iniciativa privada (Hewitt, 2009; Aguayo, 2002).

Para adquirir una canasta básica con precios controlados, los consumidores rurales deben mostrarse en una situación depauperada y en alta pobreza alimentaria. Así, la "atención" por parte del Estado justifica la visión asistencial, hacia zonas en "desventajas específicas", una vez validada la elegibilidad de los beneficiarios. La limitante al ejercicio pleno de las actividades agrícolas, desvincula a éstas de sus prácticas sustantivas. Además hay otros programas improvisados que transfieren a los "beneficiarios" la responsabilidad del diseño y administración de proyectos, a realizarse en condiciones de franca desventaja económica (Aguayo, 2002).

El desarrollo económico se esboza como una condición de individuos atomizados, movidos por la racionalidad de costo-beneficio. Pero las iniciativas gubernamentales tendrían un mejor diseño, una mejor implementación y mejores resultados, con la participación de los ciudadanos (González y Macías, 2007; Subirats, 1995, Canto, 2000, Cabrera, 2000).

Urge un interlocutor válido dentro del Estado mexicano, quegaranticela bienvenidaalas propuestas de una vida rural decorosa, cuyo contexto macroeconómico implique éxito para el sector primario, de pequeña y mediana escala (Hewitt, 2007).

\section{Datos oficiales sobre la alimentación en México y Quintana Roo, 2011}

La Secretaría de Desarrollo Social ubica áreas y hogares "pobres", diagnosticándolos vía las barreras al desarrollo social y administrando soluciones. Las unidades de ejecución son los municipios, que acceden a fondos federales por sus condiciones de marginación, pobreza y carencia de servicios e infraestructura (Escobar, 2006). Las condiciones de pobreza alimentaria nacional, así como una aproximación a los niveles municipales en el estado de Quintana Roo (ver ilustración l).

\section{Las condiciones de alimentación en Quintana Roo}

El estado de Quintana Roo posee entre 10 y 19\% de su población en situación de pobreza alimentaria (ver mapa 1), ubicándose en un nivel medio bajo respecto al nacional. Sin embargo, en el mapa 2, los datos municipales señalan dos estratos en la entidad: uno medio bajo, que considera entre un 16 y un 33\% de la población en condiciones de mediana pobreza alimentaria; y otro restante, el cualrevela que entre un 33 y un 50\% de la población se encuentra alimentariamente depauperada. En esta última zona se ubica el municipio de Felipe Carrillo Puerto, al que pertenece el ejido X-Hazil Sur y Anexos, área de estudio en esta investigación (ver tabla 1).

Según el coneval (2005), el municipio de Felipe Carrillo Puerto ocupa el cuarto lugar en la evaluación por ingresos (alimentarios, de capacidades y de patrimonio). Retomando a Escobar (2005), se observa que la alimentación es un ámbito receptor de las circunstancias políticas asociadas con la colonización y la integración. Entonces, los conocimientos y técnicas del diagnóstico 
y tratamiento de los problemas alimentarios, poseen un perfil políticamente determinado, que incide en la configuración de las realidades alimentarias locales y de las esferas mayores.

\section{El área de estudio}

El Ejido X-Hazil Sury Anexos se localiza en la provincia biótica de la península de Yucatán, posee una extensión total de 54,441 hectáreas (INEGI, 1998). Está adscrito al municipio de Felipe Carrillo Puerto y, a su vez, se conforma por tres centros de población: X-Hazil Sur, que es la cabecera ejidal; y las localidades de Uh May y Chancah Veracruz, las cuales tienen un total de 398 ejidatarios, provenientes de los centros de población mencionados (Estrada, 2005). Sus colindancias son al Norte con el ejido Felipe Carrillo Puerto, al Sur con el ejido X-Conha, además de que colinda con algunas propiedades privadas y con algunos terrenos nacionales; al Este limita con la reserva de la biosfera de Sian Ka'an ${ }^{4}$, al Oeste con propiedades privadas, terrenos nacionales y con los ejidos de Kopchen, San Antonio y X-Conha (Estrada, 2005).

Las condiciones ambientales en relación con la situación alimentaria del ejido, implican reglas culturales sobre el acceso y uso de los recursos, pero sobre todo, implican el apego a las normatividades ambientales nacionales. Su ubicación geográfica muestra un acceso desigual a los recursos, de forma que las situaciones de hambre resultan transitorias, según lo manifiesta el CONEVAL (2005) (ver ilustración 2).

\section{Los grupos domésticos campesinos y la influencia de su desarrollo en las actividades económicas}

El objetivo es analizar la situación alimentaria de los grupos domésticos mayas macehuales del ejido X-Hazil Sury Anexos, a través de sus estrategias de reproducción y adaptación sociocultural, para conocer las actividades cotidianas que realizan, al buscar satisfacer los patrones alimentarios, como una condición inherente a sus pautas culturales.

Los grupos domésticos (GDO) son los espacios sociales básicos, autoorganizados para garantizar la reproducción cotidiana y generacional; su estructura jerárquica establece roles por parentesco, género y generación, compartidos bajo criterios axiológicos en las acciones cotidianas (García y de Oliveira, 2006: 836).

Alexander Chayanov (1974) planteó que en los grupos familiares campesinos, la mano de obra es el elemento organizativo de los procesos de producción, siendo su carácter doméstico uno de los factores principales. La composición demográfica define la actividad económica y determina el destino de la fuerza de trabajo, según la disponibilidad de trabajadores en la unidad productiva. Así, el tamaño y composición influyen en la organización, tanto cuantitativa como cualitativamente.

Para comprender la composición de los GDO, Chayanov (1974) propuso analizar el ciclo de desarrollo (desde el nacimiento hasta la muerte), ubicando dos subunidades, la de los trabajadores y la de los consumidores. La posición relativa en alguna de las diferentes fases (formación, consolidación y reemplazo), permite aplicar y explicar el principio de la cooperación compleja, según la fuerza de trabajo disponible y la intensidad de las necesidades; pues la relación consumo-trabajo en la etapa de consolidación revela un incremento en la mano de obra disponible (Chayanov, 1974) (ver tabla 2).

El volumen total producido suele corresponder cuantitativamente con la composición familiar. Se incluyen todas las actividades económicas del GDO (agropecuarias, artesanales o comerciales) para cuantificarlas (Chayanov, 1974). Si bien el crecimiento y distribución por fase del ciclo doméstico, determina la distribución de las unidades por actividad económica, nunca es el GDO quien fija exclusivamente el tamaño de una unidad de explotación, debido a los factores 
exógenos que también intervienen de forma simultánea. Por ello es necesario considerarlos al analizar a los GDO (Chayanov, 1974).

En un ajuste hecho al enfoque de Chayanov, Susan Narotzky (2007) plantea que el ciclo de desarrollo deviene en "diferenciación demográfica", cuando la variable central se deriva de las unidades de consumo, más que de las de producción. Hay que enlazar esto con un contexto económico mayor, pues los GDointeractúan de forma específica (no homogénea) con el mercado y las políticas del Estado.

Este cambio (Narotzky, 2007) busca no sólo conocer una dinámica útil, sino también abordar otros elementos centrales de la vida cotidiana que no deben demeritarse tales como los conflictos y negociaciones, entre otras formas existentes de interacción social. La intención es acceder a los mecanismos de alternancia y resistencia, pero bajo una lógica ética del autoabasto.

\section{Metodología}

Se aplicó la metodología cualitativa a través de la aplicación de una entrevista semiestructurada al 12\% de los grupos domésticos del ejido X-Hazil Sur y Anexos, seleccionados aleatoriamente y considerados como estudios de caso, para obtener información sobre: 1) Tamaño, tipo y jefatura doméstica; 2) Principales actividades económicas de los/las jefas; 3) Disponibilidad y tipos de recursos productivos; 4) Relación anual de ingresos y egresos totales y; 5) Disponibilidad de alimentos conforme al ciclo anual de producción agrícola. La información se procesó con el paquete estadístico SPSS, se cruzó con otras fuentes (impresas y de campo) y con el análisis de discurso.

Los datos no fueron compilados a través de una muestra estadísticamente representativa, de forma que la extrapolación (inferencia) para el total de GDO en el ejido X-Hazil Sur y Anexos sería arbitraria. Sin embargo, los resultados obtenidos son válidos, de acuerdo con los criterios inicialmente definidos, es decir, para aquellos GDO seleccionados como estudios de caso (Arce-Ibarra, 2008; Ramsey y Schafer, 2002).

Para acceder al "ingreso global" del grupo doméstico, se consideraron elementos como las actividades para el autoabasto, el mercado (que retornan como insumos para la producción o el consumo) y los ingresos monetarios provenientes de la comercialización. Se hizo un mapeo demográfico, asociado con la utilización de la mano de obra en las labores de los GDO, de acuerdo con su morfología. Posteriormente se vinculó esto con el acceso a la tierra, para perfilar el marco que delimita la situación alimentaria.

Finalmente, se consideraron las condiciones de autoabasto, ${ }^{5}$ en torno a las prácticas productivas y de consumo de maíz en el ejido X-Hazil Sur y Anexos. Centrarse en este producto tiene como base el hecho de ser el principal componente de la dieta mesoamericana, además de que es uno de los criterios que define el modo de vida campesino (Appendini, (N/D)).

Según el censo de población de la clínica de la Secretaría de Salud (SESA), en el año 2010, el ejido X-Hazil Sur y Anexos contaba con un total de 477 familias, que se distribuyeron, en términos administrativos, en tres comunidades. De cada una se hizo una selección aleatoria de GDO, considerados como estudios de caso, cincuenta y ocho según se muestra en la tabla 3.

Para clasificareconómicamentea los GDOconsiderados como estudios de caso, se tomó la morfología como el criterio para identificar las estrategias de reproducción.

\section{Resultados}

Hacer un estudio sobre los tipos de GDO presentes en el área de estudio, responde a la necesidad de exponer el postulado de que las familias campesinas son numerosas, con arreglos domésticos de nucleares a extenso y encabezadas por hombres. En las comunidades abordadas (Chancah Veracruz, Uh-May y X-Hazil Sur) se encontraron al menos 5 tipos de arreglos domésticos: 
- Los unipersonales (grupos no familiares de una persona).

- Los monoparentales (jefe/jefa con hijos solteros).

- Los nucleares estrictos (pareja sin hijos).

- Los nucleares conyugales (pareja con hijos solteros).

- Los extensos (pareja sin hijos o con hijos viviendo con otros parientes).

En gráfica 1. Arreglos domésticos presentes en el ejido X-Hazil Sur y Anexos. Los hallazgos reflejan tipos ideales que no representan con exactitud toda la gama de combinaciones posibles en las tres comunidades estudiadas. No obstante, se observa que coexisten diversos arreglos, que despliegan varias formas de organización doméstica. Si bien se suponía una mayoría de grupos extensos, éstos representan sólo la tercera parte; siendo poco más de la mitad de carácter nuclear conyugal. Como se verá adelante, esto repercute en sus condiciones socioeconómicas.

Posteriormente se procedió a ordenar los grupos domésticos según la frecuencia respectiva de cada tipo, en su particular etapa del ciclo de desarrollo (ver tabla 4).

Según la tabla 4, la mayoría de los grupos domésticos son conyugales y se encuentran en las etapas de formación (14), consolidación (13) y de reemplazo (3), conformando 30 casos totales; otros 12 grupos tienen una composición extensa, encontrándose en las etapas de consolidación y reemplazo (6 casos de forma respectiva).

Sepresentaron 4 grupos domésticos monoparentales, hacia ambos extremos de las etapas, es decir, en formación (1) y en reemplazo (3); además se encontraron 4 grupos nucleares estrictos, todos en la etapa de reemplazo; y finalmente 4 grupos unipersonales, todos también en la etapa de reemplazo. Tras esta clasificación, se ordenaron por perfil económico, según la actividad principal desempeñada por sus jefes y jefas.
La tabla 5 muestra que la principal actividad, constante para todos los tipos de GDO sigue siendo la agricultura, como eje principal en las pautas de reproducción. Al analizar el ciclo de desarrollo de los GDO, se observó que los nucleares conyugales en formación, deben desplegar una estrategia multifuncional para poder resolver sus requerimientos económicos y alimenticios (ver gráfica 2).

Al observar el acceso a los recursos agrarios como la categoría que explica las actividades agrícolas, los GDO (ordenados por su ciclo de desarrollo) presentan una disponibilidad de tierra, que va de 0 a 3.67 hectáreas , como cantidad máxima disponible en promedio. Aquellos en reemplazo tienen mayores acervos, pues disponen de la tierra del jefe del GDOmás la del sucesor.

La tierra de cada GDO depende tanto de la mano de obra disponible para trabajarla, como de factores culturales, tales como los arreglos comunitarios en torno a su acceso y distribución; los rumbos familiares ${ }^{6}$ en el área ofrecen una cartografía social sobre el manejo territorial en el ejido de estudio (Estrada, 2005).

Como se observó en la gráfica 2, la mayor parte de los GDOdeben desplegar diferentes actividades para dar continuidad a sus estrategias de reproducción social. En este sentido, es importante considerar el dinero que proviene de los programas gubernamentales, tales como Procampo, Apoyo a Desastres Ambientales y Oportunidades; a este nivel, los aportes recibidos señalan una distribución coincidente con sus condiciones demográficas, según se muestra en la tabla 7.

Los GDO que reciben mayores cantidades de dinero por apoyos gubernamentales, son los nucleares conyugales en la fase de formación; esto se debe a que cuentan con varios miembros en etapa escolar. Sucede lo mismo en el caso de los grupos extensos, puesto que contienen infantes, jóvenes estudiantes, productores, mujeres y a uno o dos miembros adultos mayores, los cuales disponen de un particular tipo de apoyo económico dependiendo su categoría (nivel educativo, edad, producción, sexo). 
Por otra parte, si se consideran las limitantes expresadas por la gente en la localidad, los rendimientos promedio de maíz no sobrepasan, en el mejor de los casos, una tonelada, puesto que cerca del $40 \%$ de los grupos entrevistados no obtuvieron prácticamente nada de sus cosechas, según se muestra en la tabla 8.

Frente a este déficit en la producción de maíz, la gente se abastece en las tiendas locales o en la cabecera municipal. En este tenor, debe considerarse que a la semana se consume un promedio de $44 \mathrm{~kg}$ de maíz por grupo doméstico (ver tabla 9).

Existe una ligera división entre quienes cubren su auto-abasto de maíz y entre aquellos deficitarios que compran dicho cereal, según se muestra en la gráfica 3.

Si se observan los resultados de las prácticas productivas en el ejido de estudio desde la perspectiva del desarrollo, se puede mostrar que las actividades agrícolas afrontan una situación severa, al estar en franca confrontación con los procesos de conversión económica en la región y el estado, pues se ha privilegiado abiertamente al sector de servicios, por ejemplo, a través del turismo, en menoscabo del rubro primario. Lo anterior se muestra en el crecimiento que han tenido los diferentes sectores económicos en el Producto Interno Bruto del estado de Quintana Roo (ver tabla 10).

Esta promoción económica gubernamental hacia el mercado, se articula con otros procesos adversos, tales como el limitado acceso de los mayas macehuales a recursos técnicos y financieros, a espacios de mercado a través de negociaciones equitativas y, menos aún, a la capacidad para incidir en las políticas que permitan determinar otras prioridades en el actual modelo de desarrollo, el cual busca consolidar la dependencia y la desestructuración de las bases culturales que, sin duda, podrían marcar las posibilidades del propio cambio.

\section{Conclusiones}

El trabajo de campo permitió un acercamiento a las otras realidades, obteniendo como resultado una serie de elementos centrales para promover la defensa de la diferencia cultural, considerándola como una forma de valoración de las necesidades y oportunidades económicas, bajo parámetros distintos a los del mercado (Escobar, 2005).

Las morfologías de los GDO y la manera en que transitan por las diversas etapas del ciclo de desarrollo, explican parte de la dinámica de reproducción de los casos analizados. Un eje de tales manifestaciones son las redes de relaciones por criterios de parentesco, que se articulan a escala comunitaria con las formas de organización social regional, en grupos o "guardias" para cuidar la Cruz Parlante (marco ideológico de los mayas macehuales); así como en agrupaciones económicas para la producción (forestal, la cacería, la pesca y la recolección, entre otras).Todas éstas conforman pautas generadoras de diferenciaciones económicas intracomunitarias.

Si bien la importancia de la agricultura pareciera ser relativa, sistemas como la milpa y el solar son el sustento cultural para los grupos mayas macehuales y para los pueblos mesoamericanos (Appendini, N/D). En el ejido nadie vende maíz, todo se canaliza al autoabasto. Sin embargo, la gente ha comentado al respecto que los rendimientos son ahora muy bajos, debido principalmente a cambios en las condiciones climáticas (sequías y huracanes) y a que "la tierra ya no produce como antes" (comentario del rezador de Chancah Veracruz).

Es importante destacar que las comunidades mayas macehuales plasman esquemas diferenciados de interacción, bien a través de las actividades productivas (tierra, bosques, caza, pesca, recolección); bien a través de la administración de los conocimientos, conservando germoplasmas y técnicas de manejo para las patrilíneas limitadas; reservando los valores y representaciones que estructuran la organización teocrático militar, con medidas específicas de sucesión, entre otras; consolidando y readaptando así, su reproducción social. 
Observarel hecho alimentario en elárea, permitió hacer visibles las redes de relaciones que tienen implicaciones directas sobreel acceso a la tierra (dotaciones, herencias), el trabajo colectivo involucrado para hacerproducir la tierra, y las agrupaciones (parentales o no) para desplegar modelos de distribución, cotidianos y ceremoniales, domésticos y comunitarios, locales y regionales, cuya especificidad son los sistemas económico-políticos vigentes a través de una estructura patrilineal y teocrática militar.

En este sentido,la participación comunitaria permitió reconocer formas cooperativas (no necesariamente equitativas) de producción, distribución y consumo, así como los sistemas de agrupaciones para desplegar complejos marcos ideológico-ceremoniales (Santos, 2008). Estos acuerdos de colaboración esbozan las estrategias de reproducción social (Canto, 2000), sin circunscribirse exclusivamente al asistencialismo gubernamental individualizado o al mercado.

El análisis de la pobreza alimentaria a nivel local, sugiere una redefinición sustantiva de las políticas sociales desde una perspectiva integral, pues, si bien se diagnosticaron las cadenas de relaciones que conforman la pobreza multidimensional (CONEVAL), las iniciativas gubernamentales de apoyo se mueven de manera disociada en el ejido (Procampo, Oportunidades, Apoyo por Desastres). Según los datos obtenidos, la dinámica de los GDO implicó, entre otras cosas, "beneficios" sociales diferenciados.

Es urgente complementar estos objetivos específicos para que se constituyan iniciativas viables al desarrollo social, sin menoscabos económicos, sociales y ambientales. Por supuesto, elloimplicauna transformación paradigmática, con el fin de lograr nuevas aproximaciones teóricas y prácticas desde las políticas públicas, hacia las otras realidades sociales (Santos, 2002: 13, 14).

\section{Notas}

${ }^{1}$ Esta publicación ha sido posible gracias al apoyo del Fondo Institucional de Fomento Regional para el
Desarrollo Científico, Tecnológico y de Innovación (FORDECYT), del Consejo Nacional de Ciencia y Tecnología, a través del Convenio 116306: Innovación socioambiental para el desarrollo en áreas de alta pobreza y biodiversidad de la frontera sur de México.

${ }^{2}$ El Consejo Nacional de Evaluación de la Política de Desarrollo Social.

${ }^{3}$ Tratado de Libre Comercio de América del Norte.

${ }^{4}$ La Reserva de la Biosfera de Sian Ka'an fue decretada el 20 de enero de 1986; ese mismo año se integró el Programa Internacional MAB de la UNESCO. Enseguida fue catalogada como Patrimonio Mundial por la Convención del Patrimonio Mundial/UNESCO; cuenta con una superficie de 528,147 ha.

${ }^{5}$ El autoabasto es la condición en que un GDO posee tierra para cultivar, dispone de mano de obra para trabajarla y tiene dinero para adquirir insumos en la producción; o bien, si no dispone de tierra y fuerza de trabajo, cuenta con recursos monetarios para comprar maíz en cantidades y calidades que le posibiliten cubrir su necesidades de consumo (Appendini, (N/D)).

${ }^{6}$ Rumbo familiar de trabajo: extensión de tierra ubicada dentro del límite del ejido, trabajada únicamente por integrantes de una o más patrilíneas limitadas, localizadas, que mantienen entre sí relaciones de parentesco (Estrada, 2005).

\section{Agradecimientos}

Agradezco profundamente a los Doctores Federico Morales Barragán y Ronald Nigh Nielsen, por su colaboración para la elaboración de este escrito. Sus aportes teóricos y metodológicos fueron fundamentales para consolidarlo.

\section{Bibliografía}

Aguayo, Sergio, Michael, Bruce (Comp.) (2002), En busca de la seguridad perdida. Aproximaciones a la seguridad 
nacional mexicana, Segunda edición, México: Siglo XXI Editores, 417 p.

Appendini K., García B. L. et al. N/D., (2003), “Seguridad alimentaria y 'calidad' de los alimentos: iuna estrategia campesina?", en Revista Europea de Estudios Latinoamericanos y del Caribe, núm. 75, Amsterdam, Holanda, Pp. 65-83.

Arce-Ibarra, A. M. y Charles, A. T. (2008), "Inland fisheries of the Mayan Zone in Quintana Roo, Mexico: Using a combined approach to fishery assessment for data-sparse fisheries", en Fisheries Research, núm. 91, pp. 151-159.

Bartolomé, Miguel A. (1998), La dinámica social de los mayas de Yucatán. Pasado y presente de la situación colonial, México: Instituto Nacional Indigenista.

Bello, Eduardo, (2001), Milpa y madera, la organización de la producción entre mayas de Quintana Roo, Tesis de Doctorado en Antropología Social, México: Universidad Iberoamericana.

Cabrero M. Enrique (2000), "Usos y costumbres en la hechura de las políticas públicas en México. Límites de las policy sciences en contextos cultural y políticamente diferentes", en Gestión y política pública, núm. 9, vol.2, pp. 189-229.

Camou, Antonio (2001), "Estudio preliminar", en Los desafíos de la gobernabilidad, México: FLACSO, UNAM, Plaza y Valdés, pp. 15-58.

Canto S. Rodolfo (2000), "Políticas públicas. Más allá del pluralismo y la participación ciudadana", en Gestión y política pública, núm. 9, vol. 2, pp. 231-256.

Coraggio J. L. (1999), “iEs posible pensar alternativas a la política social neoliberal?", en Nueva Sociedad, núm. 164, noviembre-diciembre 1999, pp. 95-105.

Chayanov, V. Alexander (1974), La organización de la unidad económica campesina, Buenos Aires, Argentina: Ediciones Nueva Visión.

Escobar, Arturo, (2005), "El postdesarrollo como concepto y práctica social", en Daniel Mato (coord.), Políticas de economía, ambiente y sociedad en tiempos de globalización, Caracas: Facultad de Ciencias
Económicas y Sociales, Universidad Central de Venezuela, pp. 17-31.

Escobar, Arturo (2006) (en prensa), "Descentralización y política social: del centralismo al desconcierto", en http://lanic.utexas.edu/project/etext/lilas/vrp/escobar. pdf.

Estrada, L. (2005), Grupo doméstico y usos del parentesco entre los mayas macehuales del centro de Quintana Roo: el caso del ejido X-Hazil Sur y Anexos y anexos, Tesis de doctorado en Antropología social por la Universidad Iberoamericana, México.

FAO (2002), "El estado de la inseguridad alimentaria en el mundo 2002: inseguridad alimentaria, la población se ve obligada a convivir con el hambre y, teme morir de inanición", en Documentos Mixtos, Edición 4, Editor Food \& Agriculture Org.

González H., y Alejandro Macías (2007), "Vulnerabilidad alimentaria y política en México", en revista cuatrimestral Desacatos, núm. 25, Saberes y razones, México: CIESAS, septiembre-diciembre, Pp. 47-78.

Hewitt, Cynthia (2007), "Ensayo sobre los obstáculos al desarrollo rural en México. Retrospectiva y Prospectiva", en Desacatos, núm. 25, septiembreoctubre, México: Centro de Investigaciones y Estudios Superiores en Antropología Social, Distrito Federal, pp. 79-100.

Hewitt, Cynthia, (2009), "Renovación de ideas sobre el campo mexicano en época de crisis", en Conferencia magistral para el Séptimo Congreso de la Asociación Mexicana de Estudios Rurales, San Cristóbal de las Casas, México.

Martín B. Jesús (1987), De los Medios a las Mediaciones, Barcelona: Editorial Gustavo Gili.

Mauss, Marcel, (1979) (1925), "Ensayo sobre los dones. Motivo y formas del cambio en las sociedades primitivas", en Marcel Mauss, Sociología y antropología, Madrid: Tecnos.

Narotzky, S. (2003), Economía y cultura: la dialéctica de la antropología económica, Quaderns de l’Institut Català d'Antropologia, ISSN: 0211-5557, núm. 19. pp. 133-143. 
Ramsey, F.L., Schafer, D.W. (2002), The Statistical Sleuth. A Course in Methods of Data Analysis, Segunda edición, Duxbury: Thomson Learning.

Santos, B. De Sousa (2002), Renovar la teoría crítica y reinventar la emancipación social, Buenos Aires: CLACSO.

Santos, H. (2008), "Religiosidad y espacio social: una micro-región maya de Quintana Roo”, en Jiménez et al (Coord.) (2008), Los mayas contemporáneos, España: Plaza y Valdés.

Subirats Joan (1995), "Los instrumentos de las políticas, el debate público y el proceso de evaluación”, en Gestión y Política Pública, núm. 4, vol. 1, pp. 5-23.

Tuirán, Rodolfo (1993), Estructura familiar: continuidad y cambio, México: Demos, pp. 20-23.

\section{Electrónicas}

Consejo Nacional de Evaluación de la Política de Desarrollo Social (2010), El Ramo 33 en el desarrollo social en México: evaluación de ocho fondos de politica pública, México: CONEVAL, www.coneval.gob.mx/ contenido/med_pobreza/2196.xls.

Herrera, Fidel (2011), "Acuerdo Nacional contra la Pobreza”, en Periódico El Sol de México, www.oem.com. mx/elsoldemexico/, [consultado: abril 6 de 2011].

Instituto Nacional de Estadística, Geografía e Informática, (2011),www.inegi.org.mx/inegi/contenidos/ espanol/prensa/Boletines/Boletin/Comunicados/ Especiales/2011/Abril/comunica23.pdf.

Ilustración 1. Distribución nacional y de Quintana Roo sobre las condiciones de pobreza alimentaria Gráfica 2. Actividad principal por tipo de Grupo Doméstico

\begin{tabular}{|l|l|} 
Mapa 1. Población en pobreza alimentaria por estados & $\begin{array}{c}\text { Mapa 2. Población en pobreza alimentaria } \\
\text { al nivel municipal, en Quintana Roo }\end{array}$ \\
\hline
\end{tabular}




\section{Ilustración 2. Mapa del Ejido X-Hazil Sur y Anexos}
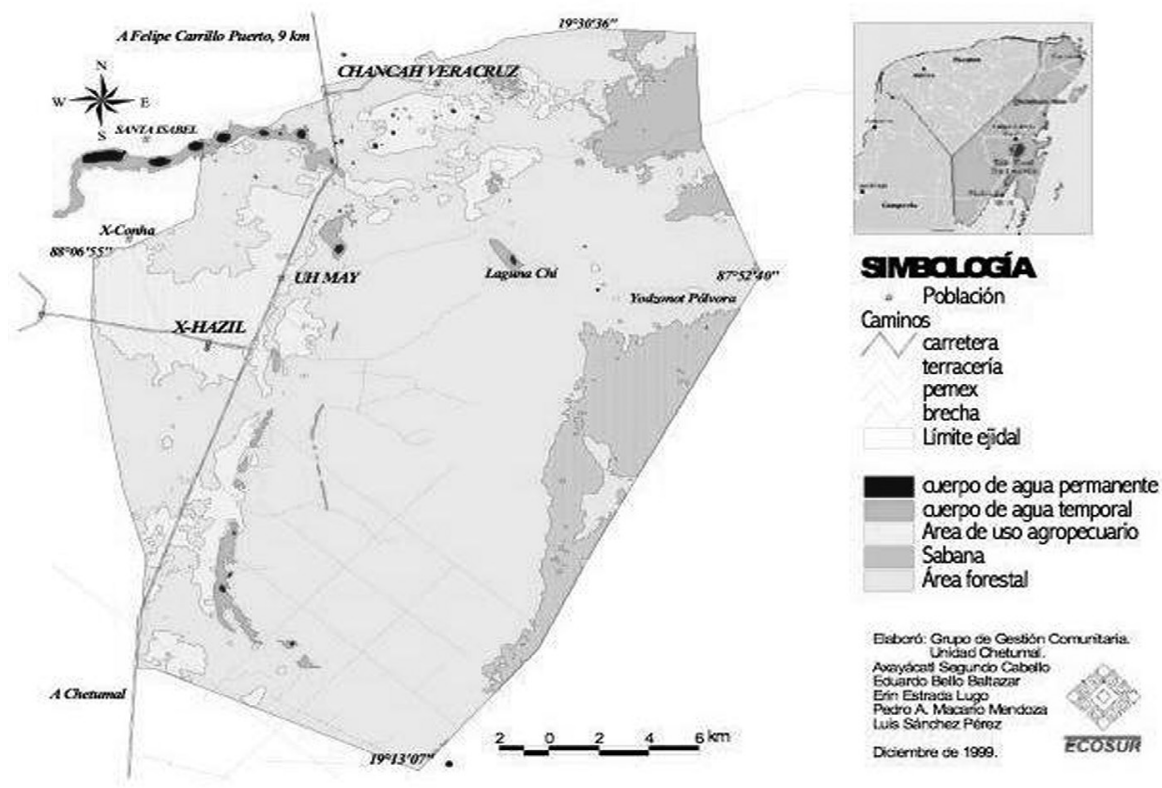

Gráfica 1. Arreglos domésticos presentes en el Ejido X-Hazil Sur y Anexos

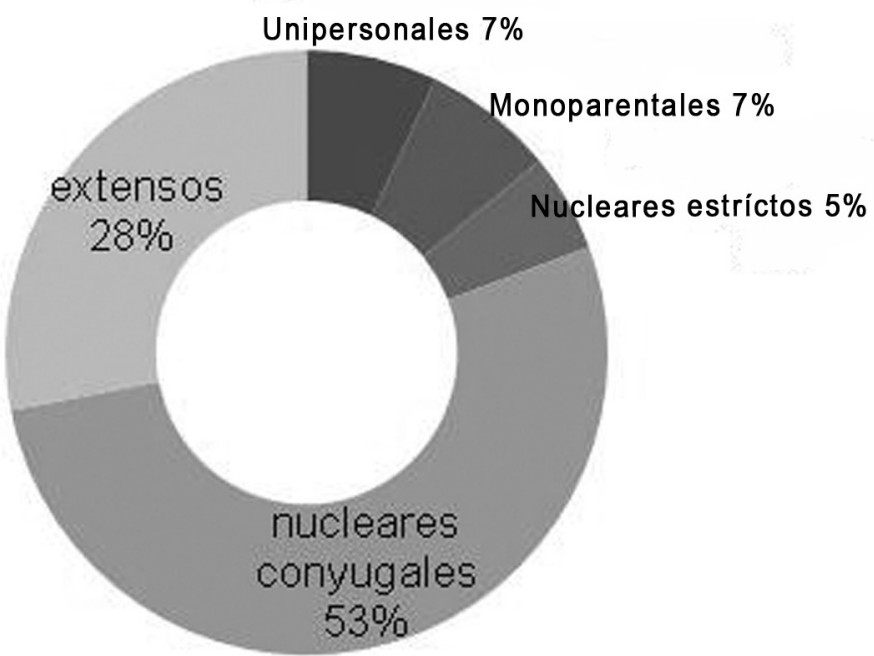


Tabla 1. Condiciones de pobreza Multidimensional, Quintana Roo

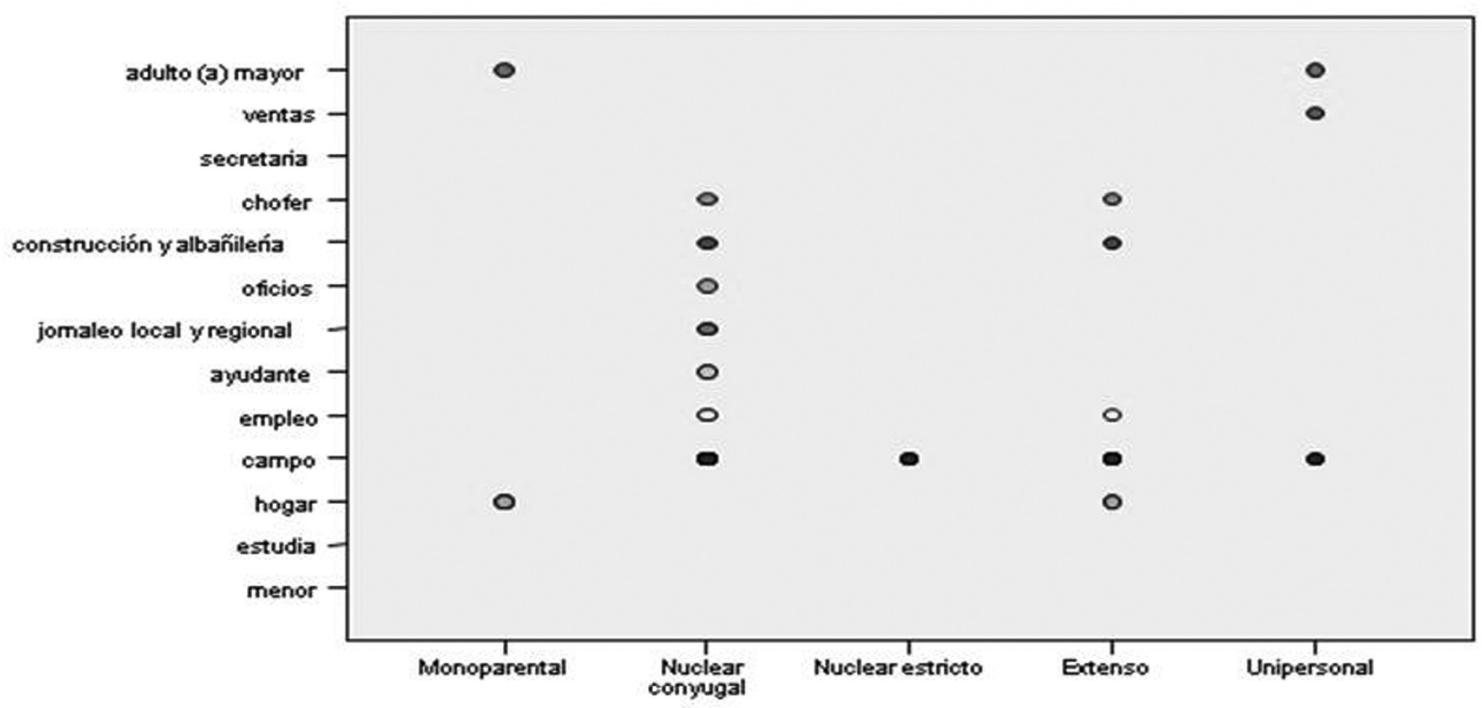

Tipo de Grupo Doméstico

Gráfica 3. Grupos domésticos por condiciones de autoabasto o semiabasto de maíz (compran maíz).

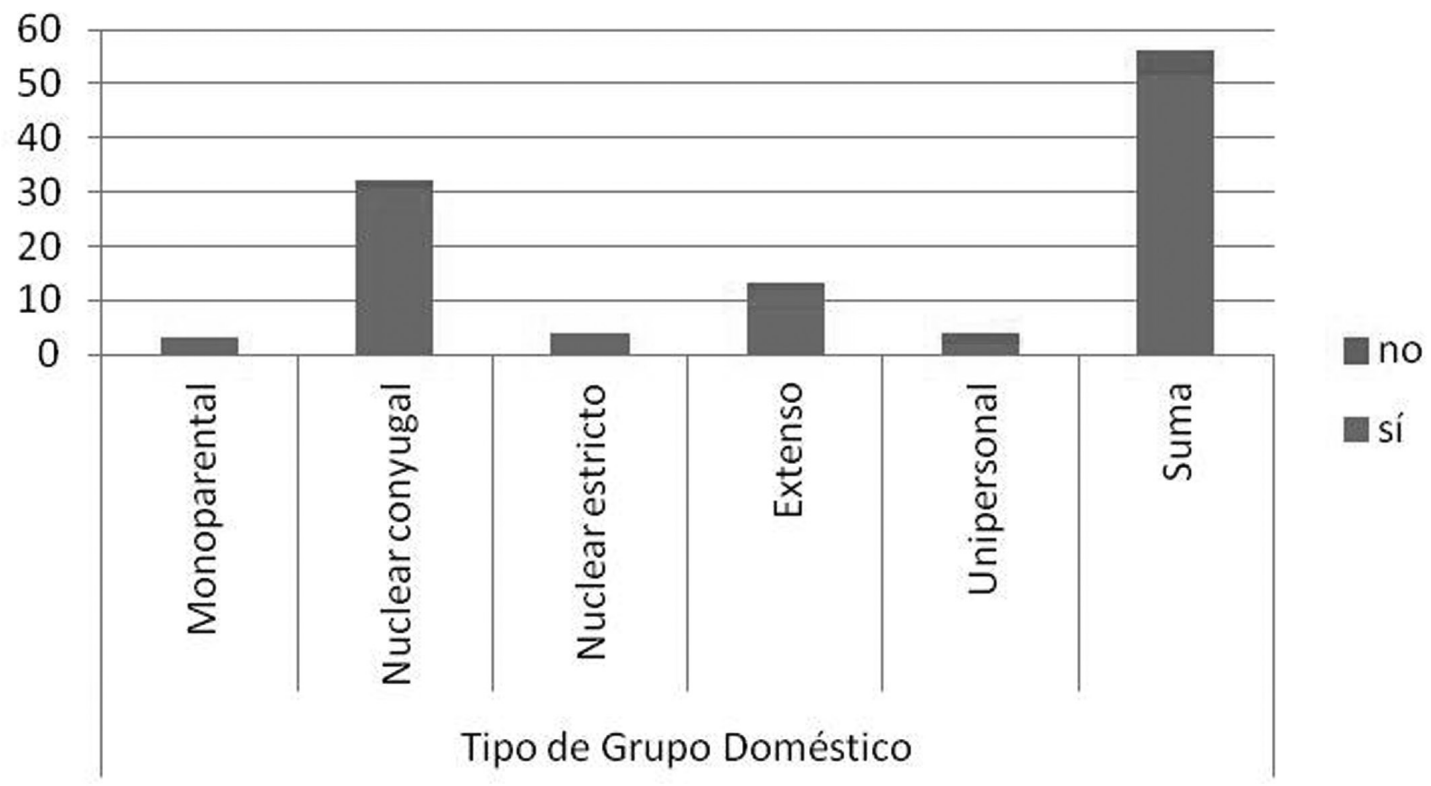




\begin{tabular}{|l||c||c||c||c||}
\hline \multicolumn{1}{|c||}{ Municipio } & Población 2000 & $\begin{array}{c}\text { Pobreza } \\
\text { alimentaria (\%) }\end{array}$ & $\begin{array}{c}\text { Pobreza de } \\
\text { capacidades (\%) }\end{array}$ & $\begin{array}{c}\text { Pobreza de } \\
\text { patrimonio (\%) }\end{array}$ \\
\hline \hline Quintana Roo & 874,963 & 23.3 & 30.4 & 52.4 \\
\hline \hline Cozumel & 60,091 & 12.5 & 19.7 & 44.7 \\
\hline Felipe Carrillo Puerto & 60,375 & 61.6 & 69.3 & 84.7 \\
\hline \hline Isla Mujeres & 11,312 & 11.5 & 16 & 32.3 \\
\hline \hline Othón P. Blanco & 208,164 & 27 & 34.6 & 57.3 \\
\hline \hline Benito Juárez & 419,816 & 13.3 & 20 & 42.8 \\
\hline \hline José María Morelos & 31,052 & 65.9 & 72.4 & 84.5 \\
\hline \hline Lázaro Cárdenas & 20,496 & 59.3 & 66.5 & 81.3 \\
\hline \hline Solidaridad & 63,657 & 20.3 & 28.7 & 54.6 \\
\hline
\end{tabular}

Fuente: www.coneval.gob.mx.

Tabla 2. Principio de cooperación compleja y ciclo de desarrollo de los grupos domésticos

\begin{tabular}{|c|c|c|c|c|}
\hline Ciclo de desarrollo & Consumidores & & Trabajadores & \\
\hline Formación & 2 & $\begin{array}{c}\text { esposa y primeros } 2 \\
\text { hijos }\end{array}$ & 1 & el jefe \\
\hline Consolidación & 3 & $\begin{array}{c}\text { esposa y } 1 \text { o } 2 \text { hijos } \\
\text { pequeños }\end{array}$ & 3 & $\begin{array}{l}\text { jefe e hijo(s) mayor(es) } \\
\text { cooperación compleja }\end{array}$ \\
\hline Reemplazo & 4 & $\begin{array}{c}\text { nuera, primeros nietos, } \\
\text { esposa }\end{array}$ & 2 & $\begin{array}{c}\text { jefe y el hijo que lo } \\
\text { reemplazará }\end{array}$ \\
\hline
\end{tabular}

Fuente: Elaboración propia con base en Chayanov, 1974. 
Tabla 3. Familias totales de X-Hazil Sur y Anexos

\begin{tabular}{||c||c|c||}
\hline \hline \multicolumn{3}{|c||}{ EJIDO X-HAZIL SUR Y ANEXOS } \\
\hline \hline Chancá Veracruz & Uh May & X-Hazil Sur \\
\hline \hline \begin{tabular}{c|c} 
Grupos domésticos totales: \\
90
\end{tabular} & Grupos domésticos totales: & Grupos domésticos totales: \\
87 & 300 \\
\hline $11(12 \%)$ & $11(12 \%)$ & $36(12 \%)$ \\
\hline
\end{tabular}

Fuente: Clínica de la Secretaría de Salud (SESA), 2010, cabecera ejidal X-Hazil Sur y Anexos.

Tabla 4. Tipo y etapa evolutiva del grupo doméstico

\begin{tabular}{|c|c|c|c|c|}
\hline \multirow[t]{2}{*}{ Tipo de Grupo Doméstico } & \multicolumn{3}{|c|}{ Etapa del Grupo Doméstico } & \multirow[t]{2}{*}{ Total } \\
\hline & Formación & Consolidación & Reemplazo & \\
\hline monoparental & 1 & 0 & 3 & 4 \\
\hline nuclear conyugal & 14 & 13 & 3 & 30 \\
\hline nuclear estricto & 0 & 0 & 4 & 4 \\
\hline extenso & 1 & 6 & 6 & 13 \\
\hline unipersonal & 0 & 0 & 4 & 4 \\
\hline Total & 16 & 19 & 20 & 55 \\
\hline
\end{tabular}

Tabla 5. Tipo de grupo doméstico y actividad principal

\begin{tabular}{|c|c|c|c|c|c|c|c|c|c|c|}
\hline \multirow{2}{*}{$\begin{array}{l}\text { Tipo de Grupo } \\
\text { Doméstico }\end{array}$} & \multicolumn{8}{|c|}{ Actividad principal } & \multirow[b]{2}{*}{$\begin{array}{c}\text { a d u I to } \\
\left(\begin{array}{c}a \\
\text { mayor }\end{array}\right)\end{array}$} & \multirow[t]{2}{*}{ Total } \\
\hline & hogar & campo & empleo & ayudante & $\begin{array}{l}\text { jornaleo } \\
\text { local y } \\
\text { regional }\end{array}$ & Albañilería & chofer & ventas & & \\
\hline Monoparental & 3 & 0 & 0 & 0 & 0 & 0 & 0 & 0 & 1 & 4 \\
\hline $\begin{array}{l}\text { Nuclear } \\
\text { conyugal }\end{array}$ & 0 & 23 & 2 & 2 & 1 & 1 & 1 & 0 & 0 & 30 \\
\hline $\begin{array}{l}\text { Nuclear } \\
\text { estricto }\end{array}$ & 0 & 4 & 0 & 0 & 0 & 0 & 0 & 0 & 0 & 4 \\
\hline Extenso & 2 & 8 & 1 & 0 & 0 & 1 & 1 & 0 & 0 & 13 \\
\hline Unipersonal & 0 & 2 & 0 & 0 & 0 & 0 & 0 & 1 & 1 & 4 \\
\hline Total & 5 & 37 & 3 & 2 & 1 & 2 & 2 & 1 & 2 & 55 \\
\hline
\end{tabular}


Tabla 6. Disponibilidad de tierra (ha) por etapa del grupo doméstico

\begin{tabular}{|c|c|c|c|c|}
\hline Etapa del Grupo Doméstico & $\begin{array}{l}\text { Tipo de Grupo } \\
\text { Doméstico }\end{array}$ & Media (Ha.) & $\mathbf{N}$ & Desv. típ. \\
\hline \multirow{3}{*}{ Formación } & nuclear conyugal & 1.6 & 15 & 1.298 \\
\hline & extenso & 0 & 1 & \\
\hline & Total & 1.5 & 16 & 1.317 \\
\hline \multirow{4}{*}{ Consolidación } & monoparental & 0 & 1 & \\
\hline & nuclear conyugal & 1.68 & 14 & 0.823 \\
\hline & extenso & 1.33 & 6 & 0.816 \\
\hline & Total & 1.5 & 21 & 0.866 \\
\hline \multirow{6}{*}{ Reemplazo } & monoparental & 2.5 & 2 & 0.707 \\
\hline & nuclear conyugal & 3.67 & 3 & 0.577 \\
\hline & nuclear estricto & 2.75 & 4 & 0.957 \\
\hline & extenso & 2.33 & 6 & 0.516 \\
\hline & unipersonal & 1 & 4 & 1.414 \\
\hline & Total & 2.37 & 19 & 1.165 \\
\hline \multirow{6}{*}{ Total } & monoparental & 1.67 & 3 & 1.528 \\
\hline & nuclear conyugal & 1.83 & 32 & 1.195 \\
\hline & nuclear estricto & 2.75 & 4 & 0.957 \\
\hline & extenso & 1.69 & 13 & 0.947 \\
\hline & unipersonal & 1 & 4 & 1.414 \\
\hline & Total & 1.79 & 56 & 1.167 \\
\hline
\end{tabular}


Tabla 7. Ingresos provenientes de programas de apoyo gubernamentales

\begin{tabular}{|c|c|c|c|c|c|}
\hline & \multicolumn{5}{|c|}{ Ingresos promedio recibidos en un ciclo anual } \\
\hline Tipo de Grupo Doméstico & Monoparental & Nuclear conyugal & Nuclear estricto & Extenso & Unipersonal \\
\hline Apoyo de procampo & 300 & 920 & 1225 & 1127 & 263 \\
\hline Apoyo oportunidades & 5540 & 8394 & 2910 & 9054 & 2370 \\
\hline Apoyo por desastres & 305 & 413 & 173 & 0 & 0 \\
\hline Total & $\$ 6,145$ & $\$ 9,727$ & $\$ 4,308$ & $\$ 10,181$ & $\$ 2,633$ \\
\hline
\end{tabular}

Tabla 8. Maíz cosechado por hectárea en el ciclo agrícola de temporal (kg)

\begin{tabular}{|c|c|c|c|}
\hline Kilogramos (Kg.) & Frecuencia & Porcentaje válido & Porcentaje acumulado \\
\hline 0 & 22 & 38 & 38 \\
\hline 10 & 1 & 2 & 40 \\
\hline 50 & 3 & 5 & 45 \\
\hline 100 & 16 & 28 & 72 \\
\hline 150 & 6 & 10 & 83 \\
\hline 200 & 2 & 3 & 86 \\
\hline 250 & 1 & 2 & 88 \\
\hline 350 & 1 & 2 & 90 \\
\hline 400 & 1 & 2 & 91 \\
\hline 500 & 1 & 2 & 93 \\
\hline 600 & 2 & 3 & 97 \\
\hline 1000 & 2 & 3 & 100 \\
\hline Casos Totales & 58 & 100 & \\
\hline
\end{tabular}


Tabla 9. Kilos de maíz comprados por semana

\begin{tabular}{|c|c|c|c|c|c|}
\hline $\begin{array}{l}\text { Tipo de Grupo } \\
\text { Doméstico }\end{array}$ & monoparental & $\begin{array}{l}\text { nuclear } \\
\text { conyugal }\end{array}$ & $\begin{array}{l}\text { nuclear } \\
\text { estricto }\end{array}$ & extenso & unipersonal \\
\hline Kg. de maíz comprado & 37 & 51 & 35 & 48 & 45 \\
\hline
\end{tabular}

Tabla 10. Participación y crecimiento promedio anual del PIB en Quintana Roo, según sectores económicos, 2009

\begin{tabular}{|c|c|}
\hline$\%$ & Sectores Económicos \\
\hline 1.1 & $\begin{array}{l}\text { Agricultura, ganadería, } \\
\text { aprovechamiento forestal, pesca y caza }\end{array}$ \\
\hline 2.1 & Minería \\
\hline 2.2 & $\begin{array}{l}\text { Electricidad, agua y suministro de gas } \\
\text { por ductos al consumidor final }\end{array}$ \\
\hline 2.3 & Construcción \\
\hline 3.1-3.3 & Industrias manufactureras \\
\hline 4.3 y 4.6 & Comercio \\
\hline 5.1 & Información en medios masivos \\
\hline 5.2 & Servicios financieros y de seguros \\
\hline 5.3 & $\begin{array}{l}\text { Servicios inmobiliarios y de alquiler de } \\
\text { bienes muebles e intangibles }\end{array}$ \\
\hline 5.4 & $\begin{array}{l}\text { Servicios profesionales, científicos y } \\
\text { técnicos }\end{array}$ \\
\hline 5.5 & Dirección de corporativos y empresas \\
\hline 5.6 & $\begin{array}{l}\text { Servicios de apoyo a los negocios y } \\
\text { manejo de desechos y servicio de } \\
\text { remediación }\end{array}$ \\
\hline 6.1 & Servicios educativos \\
\hline 6.2 & $\begin{array}{l}\text { Servicios de salud y de asistencia } \\
\text { social }\end{array}$ \\
\hline 7.1 & $\begin{array}{l}\text { Servicios de esparcimiento culturales y } \\
\text { deportivos, y otros servicios recreativos }\end{array}$ \\
\hline 7.2 & $\begin{array}{l}\text { Servicios de alojamiento temporal y de } \\
\text { preparación de alimentos y bebidas }\end{array}$ \\
\hline 8.1 & $\begin{array}{l}\text { Otros servicios excepto actividades del } \\
\text { Gobierno }\end{array}$ \\
\hline 9.3 & Actividades del Gobierno \\
\hline
\end{tabular}

Fuente:http://www.inegi.org.mx/inegi/contenidos/espanol/prensa/boletines/boletin/comunicados/especiales/20ll/abril/ comunica23.pdf. 